\title{
Evaluation of GATA4 Gene Expression in Ventricular Septal Defects Patients with Pneumonia
}

\author{
Shahram Rajaei Behbahani ${ }^{1}$, Habib Haybar (iD ${ }^{2}$, Mehdi Torabizadeh ${ }^{3}$ and Mehdi Shahrouzian (iD) ${ }^{4}{ }^{*}$ \\ ${ }^{1}$ Division of Pediatric Cardiology, Department of Pediatrics, Golestan Hospital, Ahvaz Jundishapur University of Medical Sciences, Ahvaz, Iran \\ ${ }^{2}$ Atherosclerosis Research Center, Ahvaz Jundishapur University of Medical Sciences, Ahvaz, Iran \\ ${ }^{3}$ Golestan Hospital Clinical Research Development Unit, Ahvaz Jundishapur University of Medical Sciences, Ahvaz, Iran \\ ${ }^{4}$ Department of Pediatrics, Abuzar Pediatrics Hospital, Ahvaz Jundishapur University of Medical Sciences, Ahvaz, Iran \\ "Corresponding author: Department of Pediatrics, Abuzar Pediatrics Hospital, Ahvaz Jundishapur University of Medical Sciences, Ahvaz, Iran. Email: \\ b.movahedi1360@gmail.com
}

Received 2021 January 26; Revised 2021 April 29; Accepted 2021 May 01.

\begin{abstract}
Background: GATA-binding protein 4 (GATA4) can regulate vital genes, such as troponin C, and play an important role in cardiac formation and immune response maturation.

Objectives: In this study, GATA4 gene expression was evaluated in two groups of ventricular septal defect (VSD) patients with pneumonia and healthy cases.

Methods: This case-control study evaluated GATA4 gene expression by real-time polymerase chain reaction in 80 participants and statically estimated the association between GATA4 expression and immunologic markers, such as immunoglobulin M, immunoglobulin A, mean corpuscular hemoglobin, white blood cell neutrophil, lymphocyte, and number of abortions in mothers of VSD patients with pneumonia.

Results: According to the results, there was a significant relationship between mean birth weight and neutrophil-to-lymphocyte ratio with GATA4 gene expression in the case group $(\mathrm{P}<0.05)$.

Conclusions: It can be concluded that GATA4 gene expression, especially in patients with VSD, can indicate their susceptibility to pneumonia.
\end{abstract}

Keywords: Ventricular Septal Defects, GATA4, Pneumonia, Congenital Heart Malformations, Mutation, Gene Expression

\section{Background}

The prevalence of ventricular septal defects (VSD) is about $40 \%$ of all congenital heart malformations. The diagnosis of VSD is based on a wide range of abnormalities, such as isolated defects and other congenital heart defects. The management of VSD depends on several factors, such as the size and anatomical relationship of the anomaly and patient age (1). The VSD is a hole between the lower chambers of the heart. Oxygen-rich blood is pumped from the lungs to the aorta from the left ventricle. During this process, the small amounts of blood are returned to the right ventricle and pulmonary arteries in VSD patients (2).

GATA-binding proteins are transcription factors that regulate the expression of genes and differentiation in different cell types. These proteins have a deoxyribonucleic acid binding site known as the GATA motif, recognizing conserved signature indels (i.e., an important part of the promoter of many genes). GATA4 is expressed in the adult vertebrate heart, intestinal epithelium, and gonads. During fetal development, GATA4 is expressed in the endometrium of the yolk sac and cells involved in heart formation. The evidence showed that GATA4 can regulate vital genes, such as troponin C, and heavy chain of the alphamyosin. One of the features of GATA proteins is the permanent sequencing that binds to the targeted GATA promoters.

The GATA family includes six members (i.e., GATA1 to GATA6). In addition, GATA4, GATA5, and GATA6 are highly expressed in various mesoderm and endodermal tissues, particularly in fetal and adult hearts. The GATA4 has been the most extensively studied gene with a long list of GATA4 mutations in congenital heart diseases (CHD), such as VSD, atrial septal defect, tetralogy of Fallot, and pulmonary stenosis (3). The GATA4 produces a large cluster of endothelial inflammatory response genes in liver sinusoidal endothelial cells, which are similar to viral and interferon- 
related reactions. Therefore, it can be concluded that GATA4 can play an important role in the severity of inflammation (4).

According to the literature, the association of GATA4 with VSD and inflammation has been investigated; however, the relationship between GATA4 expression and inflammation duration in VSD patients has not been investigated. Therefore, this case-control study evaluated the expression level of GATA4 in VSD patients with pneumonia in comparison to that of healthy cases. The current study also evaluated other inflammatory markers, antibodies, and risk factors to determine their associations with GATA4 expression. It is hypothesized that VSD patients with higher levels of GATA4 expression are more prone to lung infection, and GATA4 expression can be used as an indicator of susceptibility to pneumonia in VSD patients.

\section{Objectives}

This study has been performed for the first time in Ahvaz, a city in the south of Iran, and the results will provide valuable information in this area.

\section{Methods}

\subsection{Study Population}

This case-control investigation studied 40 VSD patients under 5 years of age with pneumonia in the case group and 40 healthy cases under 5 years of age without pneumonia and VSD in the control group. The age was similar between the two groups because the present study aimed to only compare VSD patients with pneumonia to healthy cases. The patients were hospitalized at Golestan Hospital in Ahvaz, Iran. The study participants were not at legal age to give informed consent; therefore, their parents gave informed consent. The study was approved by the Ethics Committee of Ahvaz Jondishapur University of Medical Sciences (AJUMS; IRAJUMS.REC.1398.291), Khuzestan Iran, and followed the ethical guidelines of Helsinki's Declaration. The variables, including immunoglobulin profiles (i.e., immunoglobulin M [IgM], immunoglobulin G [IgG], and immunoglobulin E [IgE]), C-reactive protein, erythrocyte sedimentation rate, and messenger ribonucleic acid (mRNA) expression of GATA4, were evaluated in these patients.

\subsection{Inclusion Criteria}

1) The age of under 5 years;

2) Admitted with VSD and pneumonia.

\subsection{Exclusion Criteria}

1) Over the age of 5 years;

2) Undergoing VSD surgery;

3) Genetic diseases, such as immunodeficiency and trisomy 21.

\subsection{RNA Extraction and cDNA Synthesis}

The RNA was extracted from peripheral blood using Roche mRNA Isolation kit (Roche Life Science, Germany) for blood/bone marrow in accordance with the manufacturer's instruction. Subsequently, complementary deoxyribonucleic acid (cDNA) synthesis was performed in accordance with the manufacturer's instruction using Transcriptor First Strand cDNA Synthesis kit (Roche Life Science, Germany).

\subsection{Real-Time PCR}

The sequences of specific primers for GATA4 were F: 5'-TCTTGCAATGCGGAAAGAGG-3' and R: 5'TGCCCGTAGTGAGATGACAG-3'). Moreover, the length of the amplicon was $207 \mathrm{bp}$. Semi-quantitative real-time polymerase chain reaction (PCR) was performed for the quantification of the expression levels of GATA4. The levels of GAT4 mRNAs expression were respectively normalized against glyceraldehyde-3-phosphate dehydrogenase, and the fold change was calculated using the $2^{-\Delta \Delta \mathrm{Ct}}$ formula.

\subsection{Antibody Evaluation}

Human IgM, IgG, immunoglobulin A (IgA), and IgE ELISA Antibody kits (Stemcell Technologies, Canada) were used in this study. These kits include capturing and detecting antibodies and an Ig standard. They are designed for the quantitative detection and measurement of total human Ig separately in serum, plasma, and cell culture supernatants. The assay is sandwich enzyme-linked immunosorbent assay (ELISA) in which specimens are added to ELISA plates covered by immunoglobulin-specific antibodies. This immunoglobulin is detected by adding a detection antibody conjugated to alkaline phosphatase. The addition of the chromogenic enzyme substrate pnitrophenyl phosphate causes a colored product with an intensity directly proportional to the concentration of immunoglobulin in the sample. Immunoglobulin concentration was evaluated by comparing a serial dilution of the immunoglobulin standard analyzed in parallel. 


\subsection{Statistical Analysis}

The SPSS software (version 20) was used for statistical analysis. The $t$-test and Chi-square test were utilized for quantitative and qualitative variables, respectively. Equivalent non-parametric tests were used for abnormal results. For the analysis of the data, firstly, descriptive statistical methods, including frequency distribution tables, graphs, and indices of central tendency and appropriate dispersion, were described for the studied variables. The normality of the distribution of quantitative variables was checked by the Kolmogorov-Smirnov test. The chi-square or Fisher's exact test was used to correlate the qualitative variables.

\section{Results}

This case-control study was carried out on 80 participants ( 40 cases and 40 controls). The minimum and maximum age values in the case group were 2 and 22 months, respectively. Furthermore, the minimum and maximum age values in the control group were 5 and 23 months, respectively. In addition, $40 \%$ in the case group (VSD with pneumonia) and $2.5 \%$ in the control group (without VSD and pneumonia) were under 6 months of age. In this study, $47.5 \%$ and $60 \%$ of the patients in the case and control groups were male, respectively. There was no statistically significant difference in gender between the case and control groups ( $\mathrm{P}>0.05)$.

The mean expression values of the GATA4 gene were $0.4642 \pm 0.1206$ and $0.08222 \pm 0.03063$ in the VSD and control groups, respectively. The PCR result indicated a 5.75-fold change difference between the case and control groups revealing a significant difference $(\mathrm{P}<0.0024)$ as shown in Figure 1. The neutrophil-to-lymphocyte ratios (NLRs) were $1.26 \pm 0.59$ and $0.82 \pm 0.32$ in the VSD and control groups, respectively, with a statistically significant difference observed between the studied groups $(\mathrm{P}<0.001)$. The results showed that there was a correlation between the mean of NLR and GATA4 gene expression in the case group $(\mathrm{r}=-0.33 ; \mathrm{P}<0.05)$. While increasing the mean expression of the GATA4 gene, the average NLR declined; however, there was no complete linear relationship between the two in the case group.

There was no correlation between mean NLR and GATA4 gene expression in the control group $(\mathrm{r}=0.10 ; \mathrm{P}>0.05)$. The results demonstrated that there was a correlation between mean white blood cell (WBC) and GATA4 gene expression in the case group $(\mathrm{r}=0.54 ; \mathrm{P}<0.001)$. While increasing GATA4 gene expression, WBC was also on the rise, and

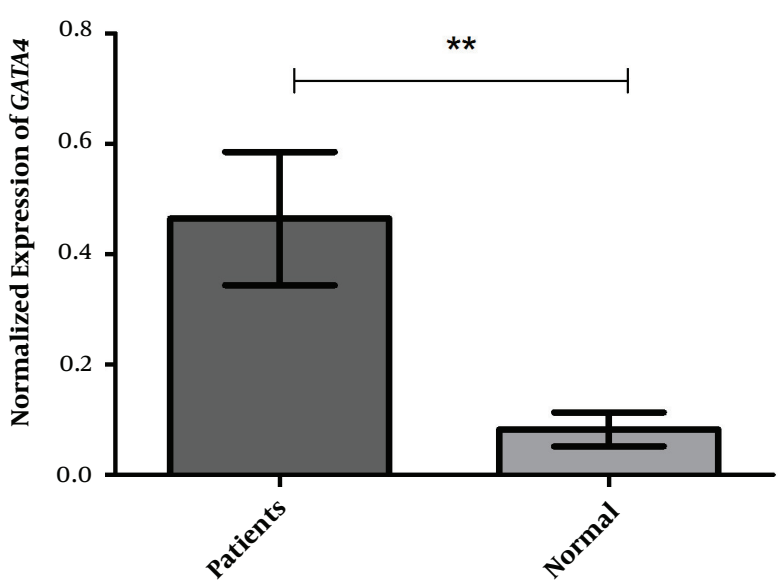

Figure 1. Normalized Expression of GATA4. This study investigated 40 cases and 40 controls. The $t$-test was utilized to compare variances, and the F, DFn, and Dfd were $15.50,14$, and 14 , respectively, indicating a statistically significant difference between the two groups $(\mathrm{P}<0.0001)$.

there was no complete linear relationship between the two in the case group. In the control group, there was a correlation between the mean of WBC and expression of the GATA4 gene $(r=0.81 ; \mathrm{P}<0.001 ;$ Figure 2$)$

The results showed a correlation between mean IgM and GATA4 gene expression in the case group $(r=-0.43$; $\mathrm{P}<0.05$ ). While increasing the mean expression of the GATA4 gene, mean IgM decreased; nonetheless, there was no complete linear relationship between the two in the case group. A similar trend was observed in the control group between IgM and GATA4 gene expression; nevertheless, there was no correlation between mean IgM and GATA4 gene expression $(\mathrm{r}=-0.32 ; \mathrm{P}>0.05)$.

The mean IgM values were $113.76 \pm 92.93$ and $60.57 \pm$ 37.79 in the case and control groups, respectively. The results revealed a statistically significant difference between the two groups in the mean of $\operatorname{IgM}(\mathrm{P}<0.001)$. While increasing the mean expression of the GATA4 gene, mean IgA decreased; however, there was no complete linear relationship between the two in the case group. A similar trend was observed in the control group between IgA and GATA4 gene expression; nonetheless, there was a correlation between mean IgA and GATA4 gene expression ( $\mathrm{r}=-0.32 ; \mathrm{P}<$ 0.05). Figure 3 illustrates the t-test of the immunoglobulins in both case and control groups. The results showed that there was a statistically significant difference between the two groups in the mean $\operatorname{IgA}(\mathrm{P}<0.001)$. 


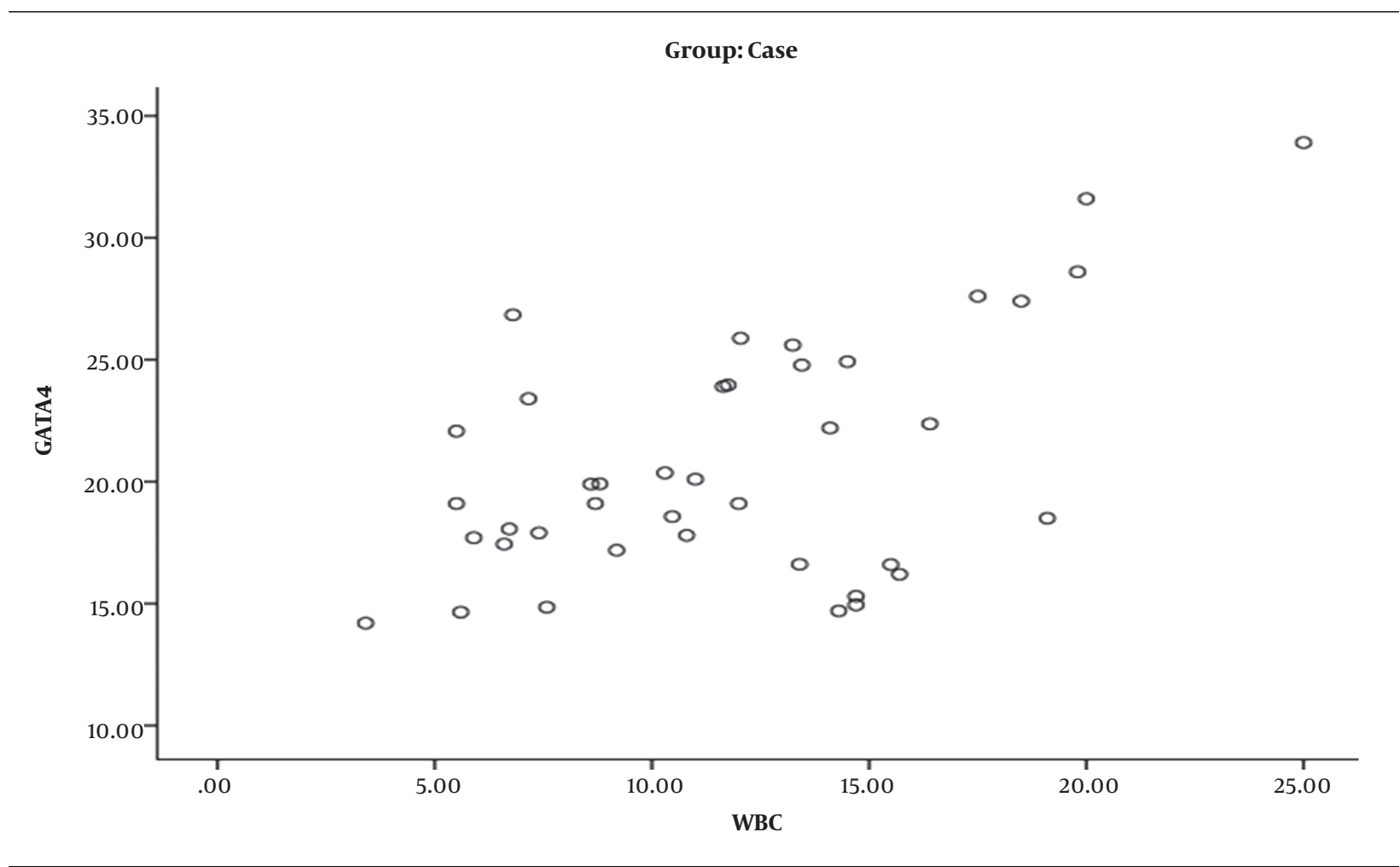

Figure 2. Results indicating correlation between mean white blood Cell and GATA4 gene expression in the case group $(\mathrm{r}=0.54 ; \mathrm{P}<0.001)$

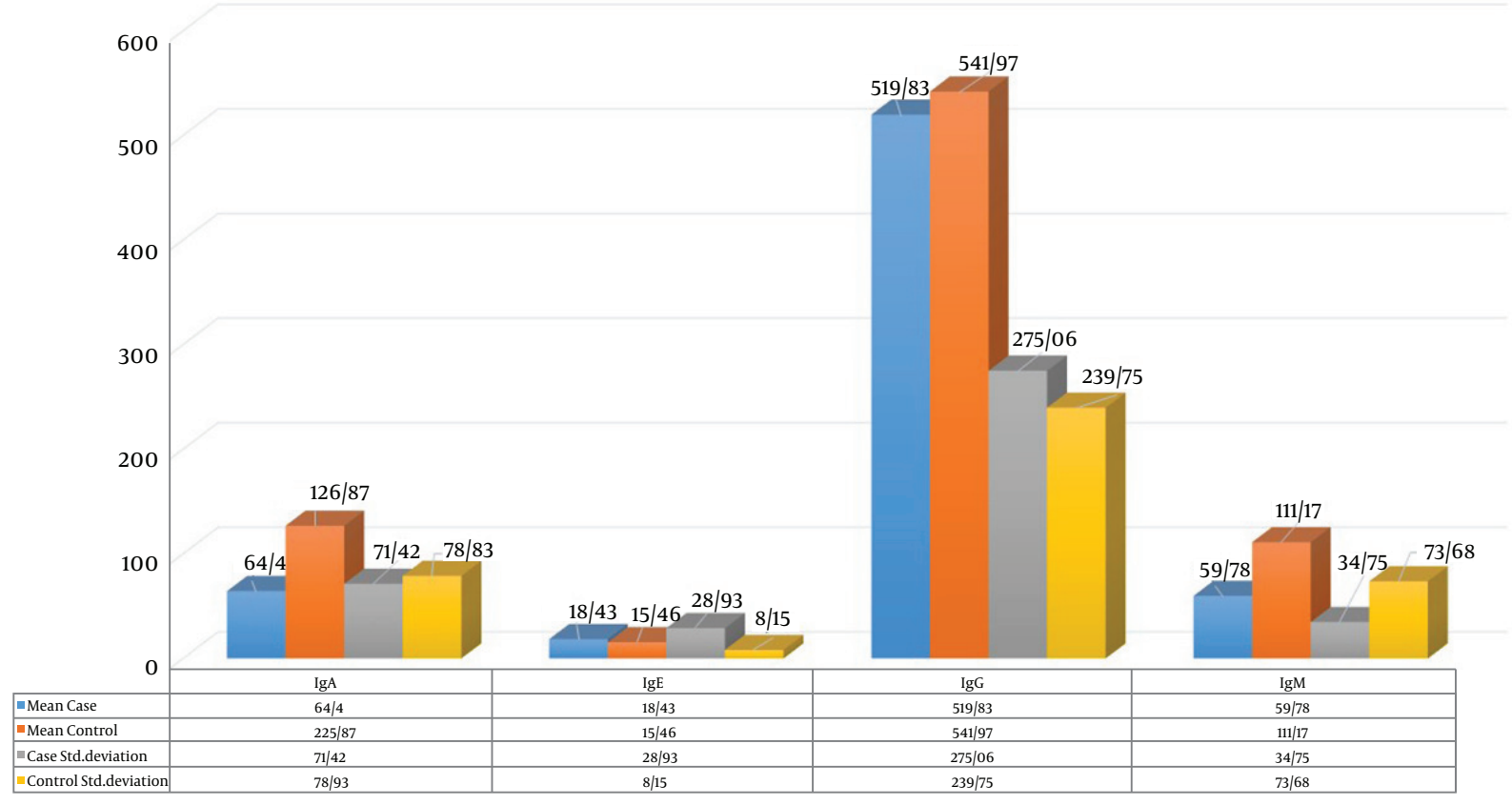

Figure 3. Mean immunoglobulin A (IgA) values were reported as 64.4 and 126.87 in the case and control groups, respectively. There was a significant difference between the two groups in the mean IgA $(\mathrm{P}<0.001)$. 


\section{Discussion}

The GATA4 is involved in the maturation of the immune system, especially innate immunity (5). Lung infection due to pneumonia is a major cause of lethality and complications in neonates. There has been limited information on the risk factors for pneumonia in children under 6 months of age. Premature delivery, CHD, antibiotic use during pregnancy, maternal overweight, daily exposure to environmental tobacco smoke before birth, and maternal smoking during pregnancy are the risk factors for neonatal pneumonia (6).

The results of a study that identified postoperative ventilator-associated pneumonia (VAP) and its risk factors in children with CHD showed chronic preoperative pneumonia, low plasma immunological protein levels, and prolonged mechanical ventilation using histamine-2 receptor blockade are closely associated with the development of VAP (7). In other words, despite preventive measures, lung infection is the most common infection after heart surgery (8). A study conducted by Yang et al. (9) on a cohort of 160 VSD patients indicated that VSD is genetically heterogeneous, and the genetic determinants for VSD remain unknown in most patients. In the aforementioned study, the entire GATA4 coding region, the gene encoding the zinc finger transcription factor essential for normal cardiac morphogenesis, was sequenced in 160 patients not associated with VSD.

A study conducted by Liang et al. (10) on mouse cells demonstrated that GATA4 and GATA6 transcription factors are the important regulators of basal and induced gene expression in a variety of heart muscles and muscle cells. The overexpression of GATA4 or GATA6 to induce cardiac hypertrophy was observed to be caused by an increase in the sarcoma network, a more than 2-fold increase in cell surface area, and a significant increase in total protein. The in vivo study of transgenic mice with a more than 2.5-fold expression of GATA4 in adult hearts has shown that hypertrophy slowly increases the heart-to-body ratio, histological features of cardiomyopathy, and activation of hypertrophyrelated genes (10). The results of a study conducted by Jia et al. (11) on GATA4 gene silencing indicated that the GATA4 transcription factor is a vital regulator of the expression of a specific gene in the heart differentiation gene and inflammatory responses. The results of the aforementioned study support the findings of the present study.

\subsection{Conclusions}

This study evaluated GATA4 gene expression in two groups of VSD patients with pneumonia and healthy sub- jects. The results of the study showed that there was a significant relationship between GATA4 gene expression in two groups with IgM, IgA, mean corpuscular hemoglobin $(\mathrm{MCH}), \mathrm{WBC}$ neutrophil, lymphocyte, and number of abortions in mothers of VSD patients with pneumonia. There was a significant relationship between mean birth weight and NLR with GATA4 gene expression in the case group. According to the results, it can be concluded that GATA4 gene expression, especially in patients with VSD, can indicate their susceptibility to pneumonia.

\section{Footnotes}

Authors' Contribution: SR wrote the constructs. HH supervised VSD section. MT supervised the role of GATA4 in immune responses. JMA supervised the genetic section. MS wrote the whole manuscript and collected the data.

Conflict of Interests: The authors declare that there is no conflict of interest.

Ethical Approval: The current study was approved by the Ethics Committee of Ahvaz Jondishapur University of Medical Sciences (code: IRAJUMS.REC.1398.291) and followed the ethical guidelines of Helsinki's Declaration.

Funding/Support: This study was supported by the Student Research Committee of Ahvaz Jondishapur University of Medical Sciences (code: IRAJUMS.REC.1398.291).

Informed Consent: Informed consent was obtained from the patients' parents.

\section{References}

1. Penny DJ, Vick G. Ventricular septal defect. Lancet. 2011;377(9771):110312. doi: 10.1016/s0140-6736(10)61339-6.

2. Hoffman JI, Rudolph AM. The natural history of ventricular septal defects in infancy. Am J Cardiol. 1965;16(5):634-53. doi: 10.1016/00029149(65)90047-0.

3. Jiang JQ, Li RG, Wang J, Liu XY, Xu YJ, Fang WY, et al. Prevalence and spectrum of GATA5 mutations associated with congenital heart disease. Int J Cardiol. 2013;165(3):570-3. doi: 10.1016/j.ijcard.2012.09.039. [PubMed: 23031282].

4. Olsavszky V, Ulbrich F, Singh S, Diett M, Sticht C, Schmid CD, et al. GATA4 and LMO3 balance angiocrine signaling and autocrine inflammatory activation by BMP2 in liver sinusoidal endothelial cells. Gene. 2017;627:491-9. doi: 10.1016/j.gene.2017.06.051. [PubMed: 28669928].

5. Santaolalla R, Abreu MT. Innate immunity in the small intestine. Curr Opin Gastroenterol. 2012;28(2):124-9. doi: 10.1097/MOG.0b013e3283506559. [PubMed: 22241076]. [PubMed Central: PMC3502878].

6. Chen $\mathrm{CH}$, Wen HJ, Chen PC, Lin SJ, Chiang TL, Hsieh IC, et al. Prenatal and postnatal risk factors for infantile pneumonia in a representative birth cohort. Epidemiol Infect. 2012;140(7):1277-85. doi: 10.1017/S0950268811001890. [PubMed: 21920066]. 
7. TAN L, ZHU X, ZHANG Z. Study on postoperative ventilation-associated pneumonia and its risk factors in children with congenital heart disease. Chin J Thorac Cardiovaesc Surg. 2001.

8. Ailawadi G, Chang HL, O'Gara PT, O'Sullivan K, Woo YJ, DeRose JJ, et al. Pneumonia after cardiac surgery: Experience of the National Institutes of Health/Canadian Institutes of Health Research Cardiothoracic Surgical Trials Network. J Thorac Cardiovasc Surg. 2017;153(6):1384-1391 e3. doi:10.1016/j.jtcvs.2016.12.055. [PubMed: 28341473]. [PubMed Central: PMC5439299].

9. Yang YQ, Li L, Wang J, Liu XY, Chen XZ, Zhang W, et al. A novel GATA4 loss-of-function mutation associated with congenital ventricular septal defect. Pediatr Cardiol. 2012;33(4):539-46.

10. Liang Q, De Windt LJ, Witt SA, Kimball TR, Markham BE, Molkentin JD. The transcription factors GATA4 and GATA6 regulate cardiomyocyte hypertrophy in vitro and in vivo. J Biol Chem. 2001;276(32):30245-53. doi:10.1074/jbc.M102174200. [PubMed: 11356841].

11. Jia W, Wu W, Yang D, Xiao C, Huang M, Long F, et al. GATA4 regulates angiogenesis and persistence of inflammation in rheumatoid arthritis. Cell Death Dis. 2018;9(5):503. doi: 10.1038/s41419-018-0570-5. [PubMed: 29717129]. [PubMed Central: PMC5931571] 